\title{
Magnetism and charge dynamics in iron pnictides
}

\author{
Z. P. Yin ${ }^{\star}$, K. Haule and G. Kotliar
}

\begin{abstract}
Unconventional superconductivity occurs in close proximity to a magnetically ordered state in many materials ${ }^{1,2}$. Uncovering the character of the proximate magnetic phase is a crucial step towards understanding the mechanism of superconductivity. Unlike the case in the cuprate superconductors, the nature of the magnetism and its underlying electronic state in the iron pnictide superconductors ${ }^{3}$ is still not well understood. Many low-energy probes such as transport ${ }^{4}$, scanning tunnelling microscopy ${ }^{5}$ and angle-resolved photoemission spectroscopy ${ }^{6}$ have measured strong in-plane anisotropy of the electronic states, but there is no consensus on its physical origin. Using a first-principles theoretical approach, we modelled the magnetic state of the $\mathrm{BaFe}_{2} \mathrm{As}_{2}$ parent compound and obtained the magnetic moment, optical conductivity and anisotropy of the electronic states, all in excellent agreement with experiments. We demonstrate that energy-dependent spin and orbital polarizations are essential features of the magnetic state in iron pnictides. Although the spin polarization is enhanced at high energy, the orbital polarization is strong only at low energy. A gain of Hund's coupling energy rather than Hubbard repulsion energy compensates the loss in kinetic energy, thereby stabilizing the lowtemperature magnetic phase.
\end{abstract}

Below the Neel temperature, of the order of $150 \mathrm{~K}$, the parent compounds of the iron pnictide superconductors remain metallic with a magnetization density oscillating in space (spin density wave, SDW). The sublattice magnetization is concentrated on iron atoms and its arrangement in space is antiferromagnetic in the $x$ direction and ferromagnetic in the $y$ direction ${ }^{7}$.

We use the combination of density functional theory and dynamical mean-field theory (DFT + DMFT; ref. 8) to theoretically study the archetypical iron pnictide compound $\mathrm{BaFe}_{2} \mathrm{As}_{2}$ in both the magnetic SDW and the paramagnetic (PM) state. The size of the theoretical magnetic moment is $0.90(5) \mu_{\mathrm{B}}$, similar to the measured moment of $0.87(3) \mu_{\mathrm{B}}$ (ref. 9), but much smaller than $1.75 \mu_{\mathrm{B}}$ (ref. 10) obtained by the local spin density approximation (LSDA) within DFT. The considerably smaller magnetic moment obtained here is due to the fact that the competing PM metallic state is a correlated metal, which contains very fast fluctuating moments in time, but no static moment. Only a small fraction of these fluctuating moments acquire a static component in the ordered state.

The onset of magnetic order has a profound impact on the electronic structure, and these changes are probed by optical spectroscopy. Figure la shows the in-plane (averaged over $x$ and $y$ directions) optical conductivity of $\mathrm{BaFe}_{2} \mathrm{As}_{2}$ in the SDW and PM states calculated by both DFT + DMFT and by standard DFT. Figure $1 \mathrm{~b}$ reproduces measured in-plane optical conductivity from refs 11 and 12 . Both theory and experiments ${ }^{11,12}$ show a reduction of the low-frequency Drude peak, which indicates a removal of a large fraction of carriers in the ordered state. Our calculation captures all the important qualitative features measured in experiments. In both PM and SDW states there is a broad peak due to interband transitions centred around $5,500 \mathrm{~cm}^{-1}$ (ref. 13). Below 2,000 $\mathrm{cm}^{-1}$ the optical conductivity of the SDW phase shows a few extra excitations appearing as peaks and shoulders centred at $1,250 \mathrm{~cm}^{-1}$ (arrow 2 in cyan), shoulder structure at about $800 \mathrm{~cm}^{-1}$ (arrow 1 in blue) and a small peak at $1,800 \mathrm{~cm}^{-1}$ (arrow 3 in green). These extra excitations appear in experiment at slightly lower energies, as seen in Fig. 1a and b.

These extra peaks and shoulders strongly depend on the polarization of light, as shown in Fig. 1c, where we plot separately the $x$ and the $y$ components of the optical conductivity. The first two excitations (blue arrow 1 and cyan arrow 2) are much more pronounced in the $x$ direction, whereas the third peak (green arrow 3) is more pronounced in the $y$ direction. The low-frequency optical conductivity is considerably larger in the $x$ direction below $1,500 \mathrm{~cm}^{-1}$, whereas it becomes larger in the $y$ direction above $1,500 \mathrm{~cm}^{-1}$. Around zero frequency, the optical conductivity of the SDW phase shows Drude-like behaviour, with Drude weight substantially smaller than in the PM phase. The theoretical value of the plasma frequencies $\omega_{\mathrm{p}, x x}, \omega_{\mathrm{p}, y y}$ and $\omega_{\mathrm{p}, z z}$ in the $x, y$ and $z$ directions are $1.19,0.95$ and $0.76 \mathrm{eV}$, respectively, much smaller than the in-plane plasma frequency of $1.60 \mathrm{eV}$ of DFT + DMFT calculation $^{13}$ and experimental measurements ${ }^{11,14}$ in the PM state. The reduction of in-plane plasma frequency in the SDW state was also observed experimentally ${ }^{11}$. The estimated resistivity ratio along the $y$ and $x$ directions $R_{y} / R_{x} \simeq \omega_{\mathrm{p}, x x}{ }^{2} / \omega_{\mathrm{p}, y y}{ }^{2}$ is about 1.57, which agrees well with recent measurements by Chu et al. ${ }^{4}$ and Tanatar and colleagues ${ }^{15}$. The anisotropy of the in-plane optical conductivity has not been studied experimentally, because of the fairly coarse spatial resolution imposed by the diffraction limit, spanning multiple magnetic domains. Near-field optics ${ }^{16}$, or techniques to prepare monodomain samples ${ }^{4}$, are promising avenues to test our prediction.

It is useful to analyse the optical conductivity at various frequency scales. The Drude weight is controlled by the Fermisurface size, and by the mass enhancement of the low-energy quasiparticles. In the SDW state, the mass enhancement is smaller (2, 1.7, 1.7 and 1.5 for the $t 2 g$ /majority, $t 2 g /$ minority, eg/majority, $e g /$ minority orbitals, respectively) than in the PM state (3 and 2 for the $t 2 g$ and $e g$ orbitals, respectively). Although the quasiparticles become lighter in the SDW phase, the Fermi-surface area is much smaller, and the latter effect dominates, resulting in a reduction of the Drude weight.

We also integrated the in-plane optical conductivity to obtain the effective kinetic energy of a low-energy model in both phases. At very low energies, the onset of magnetism results in an increase of the optical conductivity, because the SDW phase is more coherent than the correlated paramagnetic state. Consequently the very low-energy model gains kinetic energy in the ordered state. At intermediate energies, however, kinetic energy is lost as the result of the SDW gap opening on the Fermi surface. 


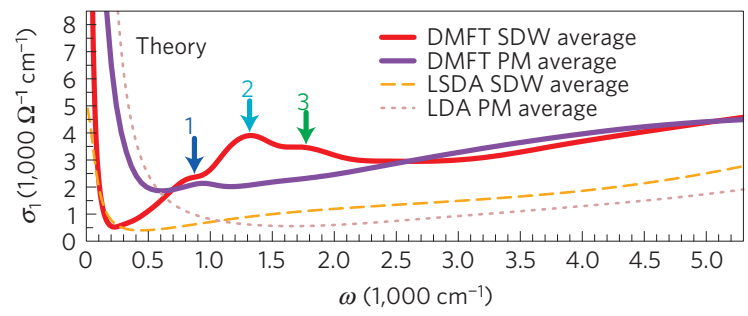

b

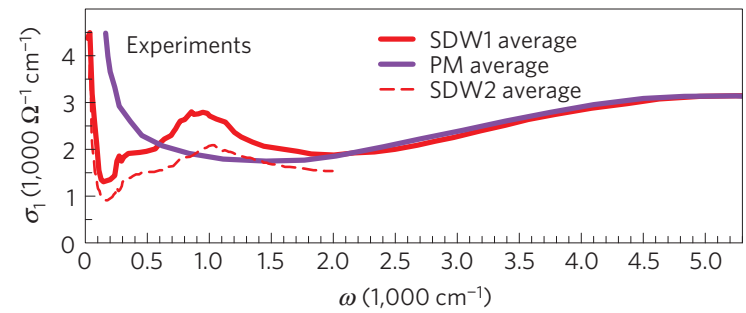

c

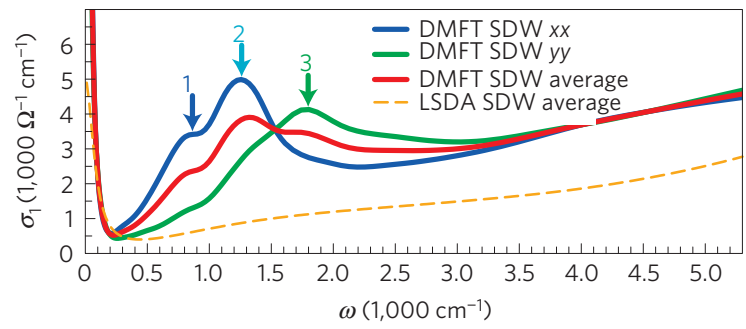

d

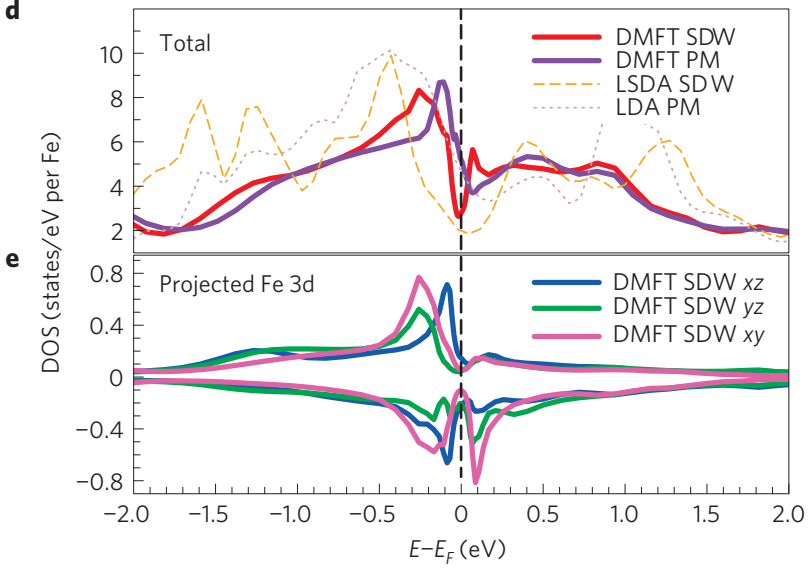

Figure 1 | Optical conductivity and DOS of $\mathrm{BaFe}_{2} \mathrm{As}_{2}$ in the SDW and PM states. a, Calculated in-plane average optical conductivity. b, Experimental in-plane average optical conductivity. Data are taken from refs 11 and 12 . c, The $x x, y y$ and in-plane average optical conductivity in the SDW state. d, Total DOS. e, The projected DOS of Fe $3 d x z, y z$ and $x y$ orbitals in the SDW state calculated by DFT + DMFT, plotting positive (negative) for majority (minority) spin.

As the optical conductivity of the PM phase is quite temperature dependent, we compare the SDW phase and PM phase at the same temperature $(T=72.5 \mathrm{~K})$, the latter being a metastable state at low temperature. Using this procedure, we find that the missing weight from opening the SDW gap is recovered only around $10,000 \mathrm{~cm}^{-1}$, a many times larger scale than the gap value. This should be contrasted with the classic weakly correlated materials, where the spectral weight is recovered immediately above the SDW gap.

We now turn to the electronic density of states (DOS). Figure 1d shows the total DOS in both the PM and SDW phases. In the SDW phase, the DFT + DMFT DOS shows a clear pseudogap on the energy scale of $0.15 \mathrm{eV}$ around the Fermi level, in good agreement with scanning tunnelling microscopy measurements ${ }^{5}$. The LSDA DOS also shows a pseudogap at the Fermi level ${ }^{17}$; however, its width is more than $0.5 \mathrm{eV}$, therefore LSDA misses the structure below $4,000 \mathrm{~cm}^{-1}$ in the optical conductivity.

The onset of the SDW phase is also accompanied by a rearrangement of the iron crystal-field states, which gives rise to orbital polarization. This polarization is uniform in space (ferroorbital polarization), as surmised by Singh ${ }^{18}$. The partial DOS of an Fe atom is shown in Fig. 1e. The minority density is given a negative sign. To extract the anisotropy of the electronic structure, we integrate the partial density of states of $x z$ and $y z$ orbitals ( $A_{x z}(\omega)$ and $\left.A_{y z}(\omega)\right)$ to obtain their occupations, and evaluate their difference $\Delta n(\Lambda)=\int_{-\Lambda}^{0}\left[A_{x z}(\omega)-A_{y z}(\omega)\right] \mathrm{d} \omega /(1 / 2) \int_{-\Lambda}^{0}\left[A_{x z}(\omega)+\right.$ $\left.\left.A_{y z}(\omega)\right] \mathrm{d} \omega\right)$. This defines the energy-dependent orbital polarization. For large cutoff $\Lambda$, the orbital polarization is close to zero for majority electrons and around 0.13 for minority electrons. At low energy, the anisotropy is enhanced to 1.23 (0.45) for majority (minority) carriers when $\Lambda$ is $0.15 \mathrm{eV}$, the size of the optical SDW gap.

The optical conductivity in the $x(y)$ direction comes primarily from excitations among $x z(y z)$ and $x y$ orbitals. The anisotropy of the partial DOS provides a natural explanation for the anisotropy of the optical conductivity; the $y z$ density of states has fewer electronic states at the Fermi level and the main peak of the $y z$ orbital is further away from the Fermi level compared with the $x z$ orbital. We notice that only the low-energy orbital polarization is large, whereas the static orbital polarization (that is, the net imbalance between the static occupations of the $x z$ and $y z$ orbitals) is very small and unimportant for the anisotropy.

In Fig. 2a, we show DFT + DMFT momentum-resolved electronic spectra $A(\mathbf{k}, \omega)$ in the SDW phase. This quantity is probed by angle-resolved photoemission spectroscopy (ARPES). Figure 2b,c compares the Fermi surface of the PM and SDW states, shown in the PM Brillouin zone.

In the PM state, the topology of the Fermi surface is very similar to DFT predictions ${ }^{19}$, with three cylinders centred at the $\Gamma$ point and two at the M point. In the SDW phase, the Fermi surfaces of the LSDA calculation (not shown) and DFT+DMFT are very different. The magnetic order reconstructs the Fermi surface into smaller more three-dimensional pockets. Out of three cylinders centred at the $\Gamma$ point, one remains in the SDW phase. This cylinder does not intersect the $\Gamma$ plane within the LSDA (not shown), but has been clearly identified in experiment. The other cylinders reconstruct into more three-dimensional pockets.

In the SDW state, there are two inequivalent directions between $\Gamma$ and $M$, here named $M$ and $M^{\prime}$, pointing along the antiferromagnetic and ferromagnetic directions of the $\mathrm{Fe}-$ Fe bonds, respectively (see Fig. 2b). Graphene-like Dirac points were recently identified by ARPES along the antiferromagnetic direction $^{6}$. Figure $2 \mathrm{a}$ shows that a crossing of two bands occurs very near the Fermi level between $\Gamma$ and $\mathrm{M}$, at three-quarters of the distance, marked by a white arrow. The crossing is below the Fermi level, hence the pocket is electron-like. In Fig. 2c we mark the same tiny pocket by blue arrows, to show that it has indeed the shape of a Dirac cone. There are two such symmetry-related Dirac cones in the $\Gamma$ plane and two in the $\mathrm{Z}$ plane. Notice that these cones appear only in the path from $\Gamma$ to $M$ (antiferromagnetic direction) and not in $\Gamma$ to $\mathrm{M}^{\prime}$.

In the most right-hand part of Fig. 2a, we overlay our results with ARPES measurements of ref. 6, to emphasize common features. The overall position of the bands is in very good agreement without any need for a shift of the Fermi level or renormalization of the bandwidth, in contrast to the common need for shifts and renormalization when comparing DFT-derived bands with ARPES.

The DFT + DMFT Fermi surface also has good agreement with the ARPES measurement in the $\mathrm{Z}$ plane in ref. 20. In particular, the red electron pockets centred at $Z$, which have a two-fold symmetry and mostly $x z$ character, were identified in ref. 20. 
a

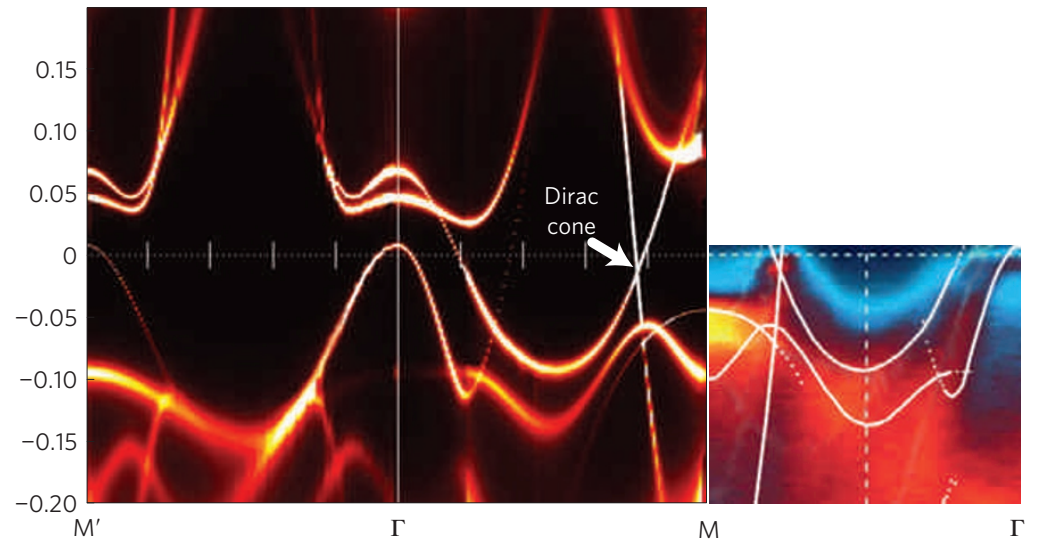

d

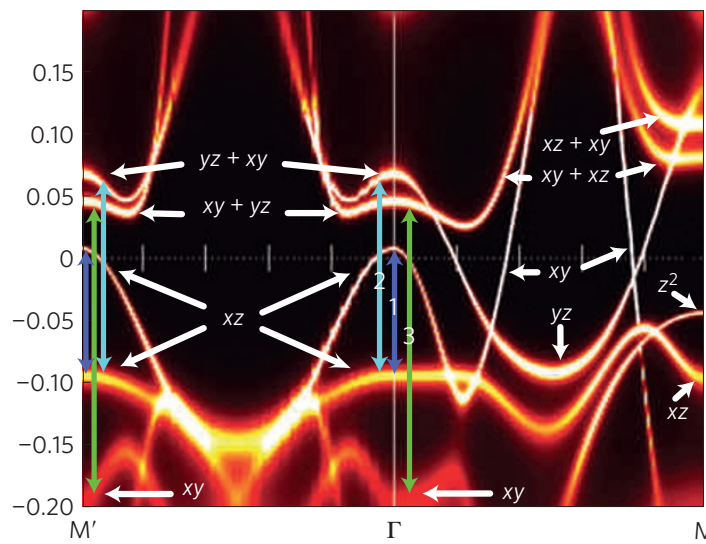

b

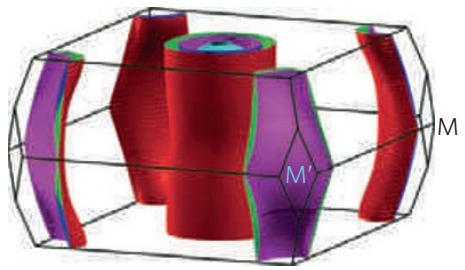

C

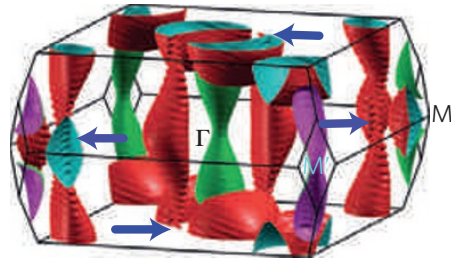

Figure 2 | ARPES and Fermi surface of $\mathbf{B a F e}_{\mathbf{2}} \mathbf{A s}_{\mathbf{2}} \cdot \mathbf{a}, A(\mathbf{k}, \omega)$ in the $\Gamma$ plane in the path $\mathrm{M}^{\prime} \rightarrow \Gamma \rightarrow M \rightarrow \Gamma$ of the SDW state. In the path $M \rightarrow \Gamma$ we overlaid ARPES data from ref. 6. b. Fermi surface in the PM state. c, Fermi surface in the SDW state, plotted in the PM Brillouin zone. Blue arrows mark the position of the Dirac cones. $\mathbf{d}, A(\mathbf{k}, \omega)$ in the SDW state with shadow bands plotted by equal intensity for clarity. Arrows mark the three types of optical transition that give rise to the three peaks in the optical conductivity.

In Fig. $2 \mathrm{~d}$ we replot the momentum-resolved electronic spectra $A(\mathbf{k}, \omega)$ without the SDW coherence factors, to enhance the shadow bands. The arrows in this graph connect the features in the electronic structure with the peaks in the optical conductivity. We mark three types of vertical transition corresponding to the three peaks in Fig. 1. The first shoulder comes primarily from transitions within the $x z$ orbital, namely between the flat band around $-0.1 \mathrm{eV}$ and the hole pocket at $\Gamma$ and $\mathrm{M}$. These transitions are between a shadow band and a non-shadow band, hence they appear only in the SDW phase. The second peak in optical conductivity, marked by a cyan arrow, comes primarily from transitions between the $x z$ and $x y$ orbitals, with some transitions between non-shadow bands only, visible also in the PM state, and some extra transitions between a shadow and non-shadow band. Finally, the third peak comes mostly from transitions between the $x y$ and $x y$ orbitals and the $x y$ and $y z$ orbitals, and mostly from transitions between a shadow and a non-shadow band.

In correlated materials, new physics, such as superconductivity, spin and orbital polarizations, emerges from the competition between Coulomb interaction and kinetic energy. A unique physical characteristic of iron arsenic materials is that the kinetic-energy loss in the SDW phase is compensated by a gain in Hund's-rule coupling energy. Comparison of the SDW and PM histograms, describing the probabilities of different iron configurations in the solid, shows that in the SDW state the high-spin states become more probable. This results in an overall gain of the Hund's-rule coupling energy of about $50 \mathrm{meV}$ per Fe. Overall kinetic energy is lost in the SDW state at about $30 \mathrm{meV}$ per $\mathrm{Fe}$, resulting in a net energy gain of about $20 \mathrm{meV}$ per Fe. This is different from the classical SDW transition, where kinetic energy is compensated by the reduction of the Hubbard correlations. Therefore, the nature of magnetism in iron pnictides is intermediate between the spin density wave of almost independent particles and the antiferromagnetic state of local moments.

The competition between kinetic and correlation energy takes different forms at different energy scales, and results in an unusual energy dependence of the spin and orbital polarizations. Spin polarization affects most strongly the electrons far below the Fermi level. For example, the exchange splitting —as determined from the frequency-dependent potential (self-energy) at high frequency-is three times larger than at zero frequency (see Supplementary Information). This high-frequency regime is governed by strong Hund'srule coupling on the iron atom, enhancing the magnetic moment.

At low energies, in the SDW state, well-defined quasiparticles form, and the residual Hund's-rule coupling between these quasiparticles is weak. To minimize the kinetic-energy loss in the spatially anisotropic SDW phase, the quasiparticles propagate mainly along the antiferromagnetic $x$ direction, hence the Drude peak is larger in the $x$ direction than in the $y$ direction. However, polarization is large only at low energy, where the quasiparticles are well formed, and the effective Hund's-rule coupling is weakest (see Supplementary Information).

Our finding that spin polarization is larger at high energy whereas the orbital polarization is most pronounced at low energies leads to definite predictions for the anisotropy of the optical conductivity and can also be tested by scanning tunnelling microscopy. 
The dichotomy of polarizations and the different characters (coherent versus incoherent) of the states close to and far from the Fermi surface is not captured by static mean-field theories. These methods can describe the physics in the low-energy range by adjusting one parameter but necessarily fail in the high-energy region. This was recently exemplified in ref. 21 , where the static method DFT $+\mathrm{U}$ with negative $U$ was used to bring the low-energy optical conductivity to better agreement with experiment than in standard DFT. To achieve this result with a static method, the magnetic moment, a high-energy quantity, has to be reduced to $0.3 \mu_{\mathrm{B}}$, substantially below its experimental value, and the resulting higher-energy optical conductivity is not in good agreement with experiment. This failure of DFT is not connected to the proximity to a quantum critical point, but has to do with the well-known fact that, in correlated situations, static mean-field theories capture correlation effects by overemphasizing the ordering tendencies and can only be remedied by a correct treatment of dynamical effects.

In a renormalization-group picture of this material, we observe a different strength of the Hund's-rule coupling at different energy scales. At high energy, Hund's-rule coupling is very strong, whereas it fades away at low energy but gives an imprint on the massive and anisotropic low-energy quasiparticles. This is central for a proper description of the magnetic phase, and is likely to be important for the mechanism of the unconventional superconductivity in these materials.

\section{Methods}

To show that the origin of the in-plane anisotropy is electronic rather than structural, we use the experimental lattice constants and internal coordinates of the paramagnetic phase ${ }^{7}$. We use the continuous-time quantum Monte Carlo as the impurity solver in its spin-rotational invariant form, and the fully charge-self-consistent version of DFT + DMFT, described in detail in ref. 22. We use the $a b$ initio-determined Coulomb interaction strength $U=5.0 \mathrm{eV}$, the Hund's coupling $J=0.7 \mathrm{eV}$ (ref. 13), and temperature $T=72.5 \mathrm{~K}$. To explore the sensitivity of the magnetic moment to the strength of the Coulomb interaction, we carried out calculations for other values of $U$ and $J$ around the ab initio values. We found that the size of the magnetic moment can be well parameterized by the simple formula $m=(0.4 \mathrm{U}+7.2 \mathrm{~J}-6.1 \mathrm{eV}) \mu_{\mathrm{B}} \mathrm{eV}^{-1}$ (see Supplementary Information). Hence, magnetization is most sensitive to the value of $J$, rather than $U$.

Received 2 July 2010; accepted 10 January 2011; published online 13 February 2011

\section{References}

1. Takabayashi, Y. et al. The disorder-free non-BCS superconductor $\mathrm{Cs}_{3} \mathrm{C}_{60}$ emerges from an antiferromagnetic insulator parent state. Science 323, 1585-1590 (2009).

2. Park, T. et al. Hidden magnetism and quantum criticality in the heavy fermion

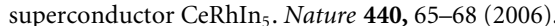

3. Kamihara, Y. et al. Iron-based layered superconductor $\mathrm{La}\left[\mathrm{O}_{1-x} \mathrm{~F}_{x}\right] \mathrm{FeAs}(x=0.05-0.12)$ with $T_{\mathrm{c}}=26 \mathrm{~K}$. J. Am. Chem. Soc. 130, 3296-3297 (2008).

4. Chu, J-H. et al. In-plane resistivity anisotropy in an underdoped iron arsenide superconductor. Science 329, 824-826 (2010).
5. Chuang, T-M. et al. Nematic electronic structure in the 'parent' state of the iron-based superconductor $\mathrm{Ca}\left(\mathrm{Fe}_{1-x} \mathrm{Co}_{x}\right)_{2} \mathrm{As}_{2}$. Science 327, 181-184 (2010).

6. Richard, P. et al. Observation of Dirac cone electronic dispersion in $\mathrm{BaFe}_{2} \mathrm{As}_{2}$. Phys. Rev. Lett. 104, 137001 (2010).

7. Rotter, M. et al. Spin-density-wave anomaly at $140 \mathrm{~K}$ in the ternary iron arsenide $\mathrm{BaFe}_{2} \mathrm{As}_{2}$. Phys. Rev. B 78, 020503(R) (2008).

8. Kotliar, G. et al. Electronic structure calculations with dynamical mean-field theory. Rev. Mod. Phys. 78, 865-951 (2006).

9. Huang, Q. et al. Neutron-diffraction measurements of magnetic order and a structural transition in the parent $\mathrm{BaFe}_{2} \mathrm{As}_{2}$ compound of FeAs-based high-temperature superconductors. Phys. Rev. Lett. 101, 257003 (2008).

10. Yin, Z. P. \& Pickett, W. E. Antiphase magnetic boundaries in iron-based superconductors: A first-principles density-functional theory study. Phys. Rev. B 80, 144522 (2009).

11. Hu, W. Z. et al. Origin of the spin density wave instability in $\mathrm{AFe}_{2} \mathrm{As}_{2}(\mathrm{~A}=\mathrm{Ba}, \mathrm{Sr})$ as revealed by optical spectroscopy. Phys. Rev. Lett. 101, 257005 (2008).

12. Nakajima, M. et al. Evolution of the optical spectrum with doping in $\mathrm{Ba}\left(\mathrm{Fe}_{1-x} \mathrm{Co}_{x}\right)_{2} \mathrm{As}_{2}$. Phys. Rev. B 81, 104528 (2010).

13. Kutepov, A. et al. Self consistent GW determination of the interaction strength: Application to the iron arsenide superconductors. Phys. Rev. B 82, 045105 (2010).

14. Chen, Z. G. et al. Optical spectroscopy of single-crystalline LaFeAsO. Phys. Rev. B 81, 100502(R) (2010).

15. Tanatar, M. A. et al. Uniaxial-strain mechanical detwinning of $\mathrm{CaFe}_{2} \mathrm{As}_{2}$ and $\mathrm{BaFe}_{2} \mathrm{As}_{2}$ crystals: Optical and transport study. Phys. Rev. B 81, 184508 (2010).

16. Qazilbash, M. M. et al. Mott transition in $\mathrm{VO}_{2}$ revealed by infrared spectroscopy and nano-imaging. Science 318, 1750-1753 (2007).

17. Yin, Z. P. et al. Electron-hole symmetry and magnetic coupling in antiferromagnetic LaFeAsO. Phys. Rev. Lett. 101, 047001 (2008).

18. Singh, R. R. P. Exchange constants and neutron spectra of iron pnictide materials. Preprint at http://arxiv.org/abs/0903.4408.

19. Singh, D. J. \& Du, M-H. Density functional study of $\mathrm{LaFeAsO}_{1-x} \mathrm{~F}_{x}$ : A low carrier density superconductor near itinerant magnetism. Phys. Rev. Lett. 100, 237003 (2008)

20. Shimojima, T. et al. Orbital-dependent modifications of electronic structure across the magnetostructural transition in $\mathrm{BaFe}_{2} \mathrm{As}_{2}$. Phys. Rev. Lett. 104, 057002 (2010).

21. Ferber, J. et al. Analysis of spin-density wave conductivity spectra of iron pnictides in the framework of density functional theory. Phys. Rev. B 82, 165102 (2010).

22. Haule, K., Yee, C-H. \& Kim, K. Dynamical mean-field theory within the full-potential methods: Electronic structure of CeIrIn ${ }_{5}, \mathrm{CeCoIn}_{5}$, and CeRhIn ${ }_{5}$. Phys. Rev. B 81, 195107 (2010).

\section{Acknowledgements}

Z.P.Y. is grateful to C-H. Yee for help at the initial stage of this project. We are grateful to D. Basov for discussions. Z.P.Y. and G.K. were supported by NSF DMR-0906943; K.H. was supported by NSF DMR-0746395 and the A. P. Sloan foundation.

\section{Author contributions}

Z.P.Y. carried out the calculations. K.H. developed the DMFT code. Z.P.Y., K.H. and G.K. analysed the results, wrote the paper and led the project.

\section{Additional information}

The authors declare no competing financial interests. Supplementary information accompanies this paper on www.nature.com/naturephysics. Reprints and permissions information is available online at http://npg.nature.com/reprintsandpermissions. Correspondence and requests for materials should be addressed to Z.P.Y. 